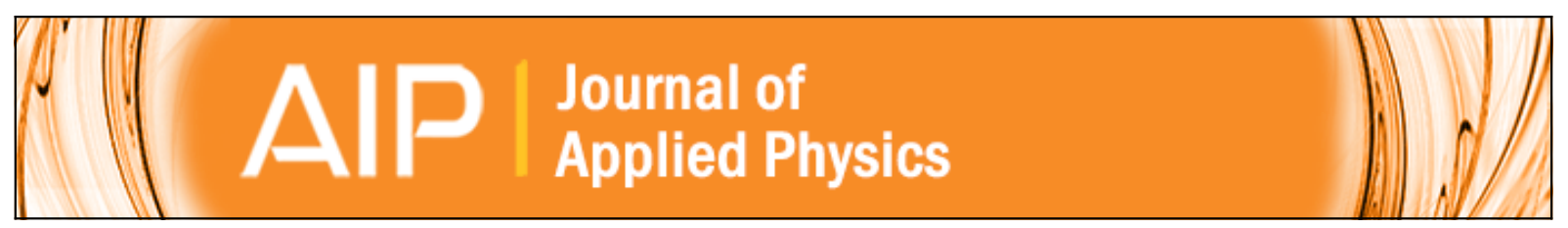

\title{
High temperature spin dynamics in linear magnetic chains, molecular rings, and segments by nuclear magnetic resonance
}

Fatemeh Adelnia, Manuel Mariani, Luca Ammannato, Andrea Caneschi, Donella Rovai, Richard Winpenny,

Grigore Timco, Maurizio Corti, Alessandro Lascialfari, and Ferdinando Borsa

Citation: Journal of Applied Physics 117, 17B308 (2015); doi: 10.1063/1.4916022

View online: http://dx.doi.org/10.1063/1.4916022

View Table of Contents: http://scitation.aip.org/content/aip/journal/jap/117/17?ver=pdfcov

Published by the AIP Publishing

\section{Articles you may be interested in}

Spin dynamics of $S=1 / 2$ Heisenberg chains with a staggered transverse field: electron spin resonance studies (Review Article)

Low Temp. Phys. 38, 819 (2012); 10.1063/1.4752094

$7 \mathrm{Li}$ nuclear magnetic resonance in the hexairon(III) antiferromagnetic molecular ring Fe6:Li

J. Appl. Phys. 95, 6879 (2004); 10.1063/1.1687225

Proton nuclear magnetic resonance investigation of the spin dynamics in cobalt based one-dimensional magnetic molecular chains

J. Appl. Phys. 93, 8749 (2003); 10.1063/1.1558597

Unconventional Heisenberg spin triangle in magnetic molecule $\{\mathrm{V} 15\}$ : A proton nuclear magnetic resonance study

J. Appl. Phys. 93, 7810 (2003); 10.1063/1.1540051

$1 \mathrm{H}$ and $23 \mathrm{Na}$ nuclear magnetic resonance study of $\mathrm{V} 6$ magnetic molecular clusters

J. Appl. Phys. 91, 7391 (2002); 10.1063/1.1448788

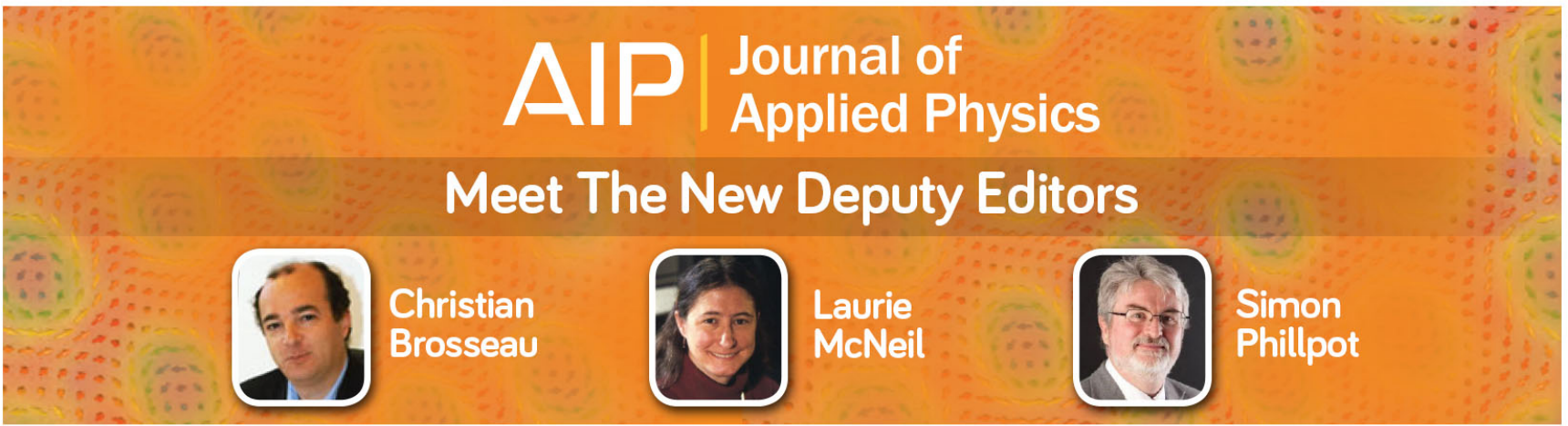




\title{
High temperature spin dynamics in linear magnetic chains, molecular rings, and segments by nuclear magnetic resonance
}

\author{
Fatemeh Adelnia, ${ }^{1,2}$ Manuel Mariani, ${ }^{3}$ Luca Ammannato, ${ }^{4}$ Andrea Caneschi, ${ }^{4}$ \\ Donella Rovai, ${ }^{4}$ Richard Winpenny, ${ }^{5}$ Grigore Timco, ${ }^{5}$ Maurizio Corti, ${ }^{2, a)}$ \\ Alessandro Lascialfari, ${ }^{1,2}$ and Ferdinando Borsa ${ }^{2}$ \\ ${ }^{1}$ Dipartimento di Fisica, Università degli Studi di Milano and INSTM, Milano, Italy \\ ${ }^{2}$ Dipartimento di Fisica, Università degli Studi di Pavia and INSTM, Pavia, Italy \\ ${ }^{3}$ Dipartimento di Fisica e Astronomia, Università di Bologna, Bologna, Italy \\ ${ }^{4}$ Dipartimento di Chimica, Università degli Studi di Firenze and INSTM, Firenze, Italy \\ ${ }^{5}$ School of Chemistry, The University of Manchester, Manchester, United Kingdom
}

(Presented 7 November 2014; received 19 September 2014; accepted 9 November 2014; published online 20 March 2015)

We present the room temperature proton nuclear magnetic resonance (NMR) nuclear spin-lattice relaxation rate (NSLR) results in two 1D spin chains: the Heisenberg antiferromagnetic (AFM) $\mathrm{Eu}(\mathrm{hfac})_{3} \mathrm{NITEt}$ and the magnetically frustrated $\mathrm{Gd}(\mathrm{hfac})_{3} \mathrm{NITEt}$. The NSLR as a function of external magnetic field can be interpreted very well in terms of high temperature spin dynamics dominated by a long time persistence of the decay of the two-spin correlation function due to the conservation of the total spin value for isotropic Heisenberg chains. The high temperature spin dynamics are also investigated in Heisenberg AFM molecular rings. In both $\mathrm{Cr}_{8}$ closed ring and in $\mathrm{Cr}_{7} \mathrm{Cd}_{\text {and }} \mathrm{Cr}_{8} \mathrm{Zn}$ open rings, i.e., model systems for a finite spin segment, an enhancement of the low frequency spectral density is found consistent with spin diffusion but the high cut-off frequency due to intermolecular anisotropic interactions prevents a detailed analysis of the spin diffusion regime. (C) 2015 AIP Publishing LLC. [http://dx.doi.org/10.1063/1.4916022]

\section{INTRODUCTION}

The problem of the spin dynamics in low-dimensional magnetic systems is of fundamental interest since, for instance, one dimensional (1D) ones are good models to obtain exact solutions and/or numerical results to be compared with the experiments, particularly in the paramagnetic phase at high temperature. In an Ising chain, there is no intrinsic spin dynamics for lack of transverse components of the spin; therefore, the dynamics are introduced ad hoc with a statistical flipping probability for the spin leading to the well-known Glauber model. ${ }^{1}$ On the other hand, for an isotropic Heisenberg chains, the spin dynamics can be calculated from the model Hamiltonian. Early numerical calculations on finite spin segments and rings indicated a persistence at long time of the decay of the two-spin correlation function $(\mathrm{CF}){ }^{2}$ This result was later confirmed by analytical solutions of a 1D Heisenberg chain, which showed a diffusive behavior at long times of the two-spin CF due to the conservation of the total spin value $\mathrm{S}^{3-5}$ Since the longtime diffusive spin motion gives a decrease as $\mathrm{t}^{-\mathrm{d} / 2}$ of the CF $(\mathrm{d}=$ dimensionality), it follows the striking result that in 1D systems there is a divergence of the spectral densities at zero frequency of the spin fluctuations. In fact, the long time slow decay (i.e., (Dt $)^{-1 / 2}$ ) of the two-spin auto-correlation function corresponds to a low-frequency enhancement of the spectral density of the spin fluctuations (i.e., $\left.(\mathrm{D} \omega)^{-1 / 2}\right)$, where $\mathrm{D}$ is the spin diffusion constant. The most direct

\footnotetext{
a) Author to whom correspondence should be addressed. Electronic mail: maurizio.corti@unipv.it.
}

experimental verification of the theory comes from the NMR spin-lattice relaxation rate (NSLR). In fact, the nuclei couple to the magnetic electrons by the hyperfine interaction and are thus sensitive to the fluctuations of the electron spins and, since the NSLR is proportional to the spectral density at the Larmor frequency, by performing NSLR measurements as a function of applied magnetic field (i.e., resonance frequency, $\omega$ ), one can directly probe the low-frequency behavior of the spectral density. Results have been published, e.g., in insulating antiferromagnetic (AFM) chains both with $\mathrm{S}=5 / 2$ (Ref. 6) and $\mathrm{S}=1 / 2,{ }^{7}$ conducting organic spin chains, ${ }^{8}$ two legs ladders ${ }^{9}$ and an $\mathrm{S}=1$ Haldane chain. ${ }^{10}$ To date, an experimental investigation of spin dynamics and spin diffusion in Heisenberg magnetic rings and short segments is still lacking. The aim of the present work is twofold: On one hand, we want to study the spin diffusion effects in two nonconventional organic 1D Heisenberg chains: the first, Eu-Et, made up of radical spins and the second, Gd-Et, a frustrated helimagnetic chain; on the other hand, we extend the investigation of spin diffusion to AFM closed molecular rings, such as $\mathrm{Cr}_{8}$ and broken rings, i.e., segments such as $\mathrm{Cr}_{7} \mathrm{Cd}$ and $\mathrm{Cr}_{8} \mathrm{Zn}$. It is worth noticing that molecular rings are a class of clusters of transition metal ions covalently bonded via superexchange bridges, embedded in a large organic molecule: ${ }^{11}$ such clusters can be synthesized in crystalline form whereby each one is magnetically independent since the intramolecular exchange interaction among the transition metal ions is dominant over the weak intermolecular, usually dipolar, magnetic interaction. In the case of rings, the magnetic ions are arranged in an almost planar circular configuration affording a perfect model system for periodic spin rings. 
${ }^{1} \mathrm{H}$ NMR measurements were performed on powder samples. In order to determine the NSLR, $1 / T_{1}$, we used a Hahn Echo pulse sequence following a saturating comb of 10-20 pulses applied at a variable delay time before the Hahn echo pulses. The length of the $\pi / 2$ pulse was in the range of 2.3-3.1 $\mu \mathrm{s}$. The recovery curve was found to be exponential, in spite of the fact that there are many non equivalent proton sites and that the systems is in form of powders, because the spin-lattice relaxation time $T_{1}$ is much longer than the spin-spin relaxation time $\mathrm{T}_{2}$, thus allowing the system to reach a common spin temperature before it relaxes to the lattice temperature.

\section{RESULTS IN 1D HEISENBERG CHAINS}

The two organic magnetic chains investigated, $\mathrm{M}(\mathrm{hfac})_{3}$ NITEt, with $\mathrm{M}=\mathrm{Eu}$ and $\mathrm{Gd}$, are isomorphous and isostructural. The coordination number for the magnetic centre is eight counting the two oxygen atoms of the two nitronylnitroxides and the six oxygen atoms of the three (hfac) moieties. The bridges of the metal ions are composed of NITEt characterized by two $\mathrm{N}-\mathrm{O}$ groups which share one electron: This ensures a strong transmission of the interaction along the chain. The Eu(III) ion is diamagnetic (at room temperature and below), and thus, the Eu-Et chain is a chain of isotropic radical $\mathrm{s}=1 / 2$ spins with $\mathrm{AFM}$ super-exchange interaction. In the Gd-Et chain there is an alternation of $\mathrm{Gd}(\mathrm{III})$ ions with spin $\mathrm{s}=7 / 2$ and of $\mathrm{s}=1 / 2$ radical spins. The nearest neighbour (nn) interaction between a Gd and a radical spin is FM, while the next nearest neighbour (nnn) interaction between $\mathrm{Gd}$ spins and between radical spins is AFM, leading to a fully frustrated chain at very low temperature with a rich phase diagram fulfilling the so-called "Villain's conjecture." 12 The exchange interactions $\mathrm{J}$ are, in all cases, of the order of $10 \mathrm{~K}$ or less, thus, the measurements performed at $\mathrm{T} \sim 300 \mathrm{~K}$ for Eu-Et and at $100 \mathrm{~K}$ for Gd-Et give information about the high temperature spin dynamics. The details of the synthesis, structure, and magnetic properties are described elsewhere. ${ }^{13,14}$ The experimental results for Eu-Et and Gd-Et are presented in Fig. 1. In the weakcollision approach, $\mathrm{T}_{1}{ }^{-1}$ can be expressed as ${ }^{15}$

$$
\mathrm{T}_{1}^{-1} \propto \Sigma_{\mathrm{ij}} \alpha_{\mathrm{ij}} \mathrm{j}_{ \pm}^{\mathrm{ij}}\left(\omega_{\mathrm{e}}\right)+\Sigma_{\mathrm{ij}} \beta_{\mathrm{ij}} \mathrm{j}_{\mathrm{Z}}^{\mathrm{ij}}\left(\omega_{\mathrm{L}}\right),
$$

where $i$ and $j$ are the number of the electronic spins, $\omega_{\mathrm{e}}$ and $\omega_{\mathrm{L}}$ are the electronic and nuclear Larmor frequencies, respectively, $\alpha_{\mathrm{ij}}$ and $\beta_{\mathrm{ij}}$ are geometrical factors related to the nuclear-electron hyperfine interaction, and $\mathrm{J}_{ \pm, z}$ are the transverse and longitudinal spectral densities of the spin fluctuations. For an isotropic Heisenberg system, the transverse and longitudinal spin fluctuations are assumed to be the same. In Eq. (1), $\mathrm{J}_{ \pm, \mathrm{z}}{ }_{\mathrm{ij}}^{\mathrm{ij}}(\omega)$ can be expressed by the Fourier transform (FT) of the two spin CF

$$
\mathrm{J}_{ \pm, \mathrm{z}}^{\mathrm{ij}}(\omega)=\int \mathrm{G}_{\mathrm{ij}}(\mathrm{r}, \mathrm{t}) \exp (\mathrm{i} \omega \mathrm{t}) \mathrm{dt} .
$$

In presence of a diffusive behavior the long time (small frequency) decay of the auto-correlation function $(r=0)$ can be
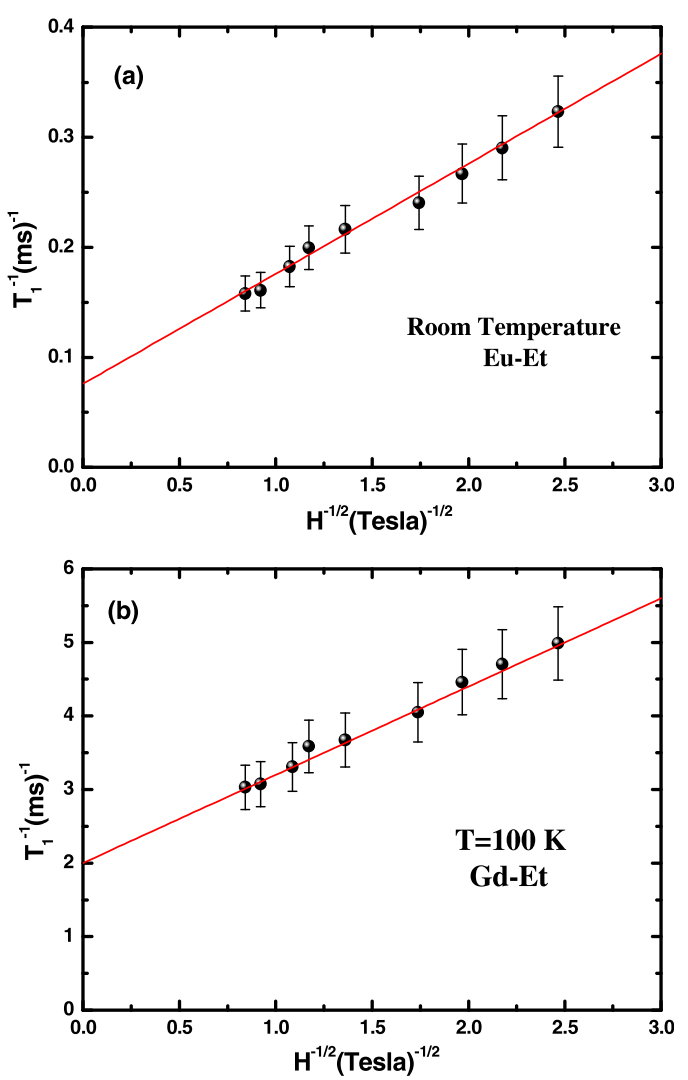

FIG. 1. Proton NSLR plotted as a function of the inverse square root of the applied magnetic field for the two 1D chains (a) Eu-Et and (b) Gd-Et. The straight lines are theoretical curves discussed in the text.

modelled (at long times the pair-correlation tends to coincide with the auto-correlation) as ${ }^{6}$

$$
\mathrm{G}_{\mathrm{ij}}(\mathrm{t})=\left\langle\mathrm{S}_{\mathrm{i}}(\mathrm{t}) \mathrm{S}_{\mathrm{j}}(0)\right\rangle=(4 \pi \mathrm{Dt})^{-1 / 2} \exp \left(-\omega_{\mathrm{c}} \mathrm{t}\right),
$$

where the first term in Eq. (3) represents the long time diffusive behavior, while the exponential decay is a phenomenological cut-off term which is introduced to simulate the effect of the anisotropic intrachain interactions in the spin Hamiltonian which can interrupt the 1D diffusive behavior by allowing spin-lattice coupling that does not conserve the total spin value $S$ and/or interchain interactions which turn the slow $1 \mathrm{D}$ spin diffusion into a much faster $3 \mathrm{D}$ decay. ${ }^{6}$ finds 6,10

By replacing Eq. (3) into Eqs. (2) and (1) one finally

$$
1 / \mathrm{T}_{1}=\mathrm{P}\left\{\left[\left(\mathrm{H}_{\mathrm{c}}^{2}+\mathrm{H}^{2}\right)^{1 / 2}+\mathrm{H}_{\mathrm{c}}\right] /\left[\mathrm{H}_{\mathrm{c}}^{2}+\mathrm{H}^{2}\right]\right\}^{1 / 2}+\mathrm{Q} .
$$

The constant Q represents the contribution due to the spectral density of the fast decaying CF at short times. This contribution becomes frequency independent at frequencies smaller than the exchange frequency defined by Eq. (6) below, which determines the initial fast decay of the $\mathrm{CF}$ (the nuclear/electron Larmor frequency is indeed smaller than the exchange frequency). The first term in Eq. (4) arises from the spectral density of the CF defined by Eq. (3), and it contains the desired information about spin diffusion and cut-off effects. The cut-off field has been introduced in place of the cut-off frequency for convenience in the presentation of the 
data whereby $\omega_{\mathrm{c}}=\gamma_{\mathrm{e}} \mathrm{H}_{\mathrm{c}}$ with $\gamma_{\mathrm{e}}=1.75 \times 10^{7} \mathrm{rad} \mathrm{Hz} / \mathrm{G}$, the electron gyromagnetic ratio. The constant $\mathrm{P}$ contains the information about spin diffusion constant $\mathrm{D}$ (in $\mathrm{rad} \mathrm{Hz}$ ), i.e., $\mathrm{P}=C / 2 \pi\left(2 \mathrm{D} \gamma_{\mathrm{e}}\right)^{1 / 2}$, where $C$ is the average square of the hyperfine interaction between nuclei and electrons in units of $(\mathrm{rad} \mathrm{Hz})^{2}$. If the cut-off effects are negligible in the field (frequency) range investigated one can set $\mathrm{H}_{\mathrm{c}}=0$ in Eq. (4) to obtain the expression which defines clearly the long time 1D spin diffusion effect

$$
1 / \mathrm{T}_{1}=\mathrm{PH}^{-1 / 2}+\mathrm{Q} .
$$

The experimental results in Fig. 1 are fitted well by Eq. (5) with $\mathrm{P}=0.1\left(\mathrm{~T}^{1 / 2} \mathrm{~m} \mathrm{~s}^{-1}\right)$ and $\mathrm{Q}=0.076\left(\mathrm{~m} \mathrm{~s}^{-1}\right)$ for Eu-Et and $\mathrm{P}=1.2\left(\mathrm{~T}^{1 / 2} \mathrm{~m} \mathrm{~s}^{-1}\right)$ and $\mathrm{Q}=2\left(\mathrm{~m} \mathrm{~s}^{-1}\right)$ for Gd-Et. This indicates that the spin dynamics in both magnetic chains are dominated, in the field range investigated, by $1 \mathrm{D}$ spin diffusion. The cut-off field must be less than $\mathrm{H}_{\mathrm{c}} \approx 0.1 \mathrm{~T}$ $\left(\omega_{\mathrm{c}}=1.75 \times 10^{10} \mathrm{rad} \mathrm{Hz}\right)$ indicating a very small intrachain anisotropy and negligible interchain interaction. Since the two chains are isomorphous, the value of the interaction constant $C$ defined above must be the same and thus from the measured values of the constant $\mathrm{P}$ one derives that the ratio of the diffusion constant for the two chains is $\mathrm{D}_{\mathrm{Eu}} / \mathrm{D}_{\mathrm{Gd}}$ $=\left(\mathrm{P}_{\mathrm{Gd}} / \mathrm{P}_{\mathrm{Eu}}\right)^{2}=144$. The implications of this result are discussed in the conclusions.

\section{RESULTS IN CLOSED AND BROKEN MOLECULAR RINGS}

The problem of the high temperature $\left(T \gg J / k_{B}\right)$ spin dynamics in 1D periodic structures like closed rings made up of Heisenberg coupled spins was investigated theoretically previously. Early numerical calculations indicated an anomalous enhancement of the spectral density of the $\mathrm{CF}$ at low frequency. ${ }^{2}$ This clearly follows from a slow decay of the $\mathrm{CF}$ due to the conservation of the total spin $\mathrm{S}$ value in a Heisenberg system. While in a $1 \mathrm{D}$ chain, the $\mathrm{CF}$ is predicted to decay diffusively at long times, as discussed in the in section II, in a periodic structure, the theoretical result is different. Several theories all lead to the conclusion that in closed rings composed of $\mathrm{N}$ spins the auto-CF decays rapidly at short times due to the exchange interaction $\mathrm{J}$ until it reaches a constant value of $1 / \mathrm{N}$ at long times, i.e., for $\mathrm{t} \gg \omega_{\mathrm{e}}{ }^{-1} \approx \mathrm{J}^{-1} \cdot{ }^{16-18}$ A simple model, which incorporates the above theoretical predictions, is obtained by assuming for the long time decay of the two spin CF in Eq. (3) the product of a constant term times an exponential cut-off function which, just like for 1D spin diffusion, represents the decay of the CF due to anisotropic intramolecular interactions and intermolecular interactions. The short time decay of the CF can be modelled with an exponential decay driven by the Moriya ${ }^{15}$ exchange frequency

$$
\omega_{\mathrm{e}}=(2 \pi \mathrm{J} / \mathrm{h})[8 / 3 \mathrm{zS}(\mathrm{S}+1)]^{1 / 2},
$$

where $\mathrm{z}$ is the number of neighbors exchange coupled to a given spin and $\mathrm{J}$ is the corresponding exchange coupling constant. By combining the CF's in Eqs. (2) and (3), finally one can write as follows: ${ }^{19}$

$$
1 / \mathrm{T}_{1}=\mathrm{A} /\left(1+\left(\mathrm{H} / \mathrm{H}_{\mathrm{c}}\right)^{2}\right)+\mathrm{C}\left(\mathrm{ms}^{-1}\right),
$$

where the magnetic field $\mathrm{H}$ is expressed in Tesla with the cut-off field $\mathrm{H}_{\mathrm{c}}=\omega_{\mathrm{c}} / \gamma_{\mathrm{e}}$ and the constant $\mathrm{C}$ depends on the spectral density of the short time fast decaying CF due to the exchange frequency $\omega_{\mathrm{e}}$ just like the constant $\mathrm{Q}$ in Eq. (4).

Equation (7) was used to fit successfully the field dependence of the proton NSLR in a number of closed molecular rings. ${ }^{19,20}$ The aim of the present investigation is to establish if the long time diffusive behavior of the CF observed in 1D Heisenberg chains can also be observed in open rings, which should correspond to 1D finite spin segments. For this purpose, we compare the previous results in closed ring $\mathrm{Cr}_{8}$ with the results in broken ring $\mathrm{Cr}_{7} \mathrm{Cd}$, where a magnetic $\mathrm{Cr}$ ion is replaced by a diamagnetic $\mathrm{Cd}$ ion and in a new kind of ring, $\mathrm{Cr}_{8} \mathrm{Zn}$, where the eight $\mathrm{Cr}$ moments are interrupted by a $\mathrm{Zn}$ diamagnetic ion, thus forming a finite spin segment. ${ }^{21}$ The experimental results obtained at room temperature for the three rings are plotted in Fig. 2. The plot as a function of $\mathrm{H}^{-1 / 2}$ allows one to see if there is a field range in which one can detect the 1D spin diffusion behaviour. The dashed straight lines correspond to the spin diffusion result given by Eq. (5). For $\mathrm{Cr}_{8}$ and $\mathrm{Cr}_{7} \mathrm{Cd}$, the straight lines have negative intercepts (negative $\mathrm{Q}$ constant in Eq. (5)). This is an unphysical result, which indicates that in $\mathrm{Cr}_{8}$ and in $\mathrm{Cr}_{7} \mathrm{Cd}$ no spin diffusion can be detected. On the other hand, in $\mathrm{Cr}_{8} \mathrm{Zn}$ it appears that there is a limited range at high fields where spin diffusion could be present. The deviation from the straight line at lower fields is due to cut-off effects. The data in Fig. 2 can actually be fitted over the whole field range either by Eq. (4) or by Eq. (7). The data in $\mathrm{Cr}_{8} \mathrm{Zn}$ can be fitted best with Eq. (4), with the fitting parameters: $\mathrm{P}=3$ $\left(\mathrm{T}^{1 / 2} \mathrm{~m} \mathrm{~s}^{-1}\right), \mathrm{Q}=0.7 \mathrm{~m} \mathrm{~s}^{-1}$, and $\mathrm{H}_{\mathrm{c}}=3.5 \mathrm{~T}$.

The data in $\mathrm{Cr}_{8}$ and $\mathrm{Cr}_{7} \mathrm{Cd}$ can be fitted best with Eq. (7) with parameters: $\mathrm{A}=2.9 \mathrm{~m} \mathrm{~s}^{-1}, \mathrm{H}_{\mathrm{c}}=9 \mathrm{~T}, \mathrm{C}=0.5 \mathrm{~m} \mathrm{~s}^{-1}$ for $\mathrm{Cr}_{8}$; and $\mathrm{A}=4.25 \mathrm{~m} \mathrm{~s}^{-1}, \mathrm{H}_{\mathrm{c}}=7 \mathrm{~T}, \mathrm{C}=0.3 \mathrm{~m} \mathrm{~s}^{-1}$ for $\mathrm{Cr}_{7} \mathrm{Cd}$. The corresponding fitting curves are shown in Fig. 2. In all three cases, the cut-off field is more than an order of magnitude larger than the one estimated for the Heisenberg chains in Fig. 1.

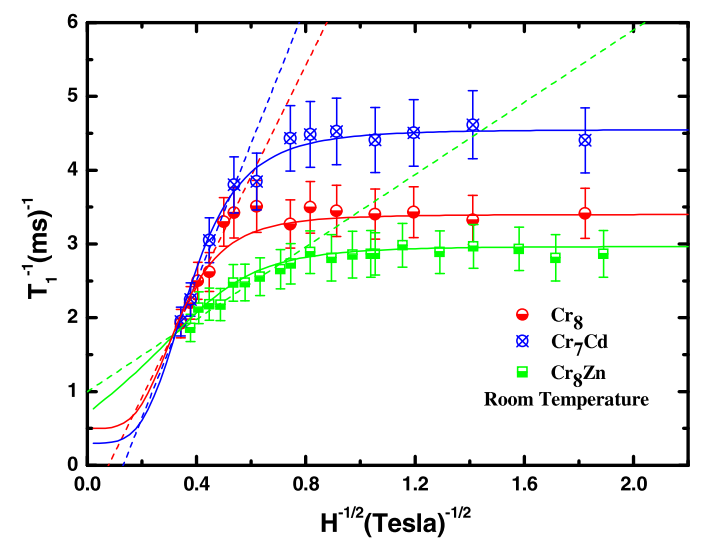

FIG. 2. Proton NSLR plotted as a function of the inverse square root of the applied magnetic field for three molecular rings. The full curves are fits according to Eq. (4) or Eq. (7). The dashed straight lines are the limiting behavior of Eq. (4) for $\mathrm{H}_{\mathrm{c}}=0$, i.e., Eq. (5). 


\section{SUMMARY AND CONCLUSIONS}

We investigated the high-temperature behavior of lowdimensional magnetic systems. In both the two 1D Heisenberg chains studied, we found very clear evidence for a slow diffusive decay of the CF due to spin diffusion. Both systems have very small anisotropy, and this allows the slow decay of the CF to persist up to long times. The time range over which the spin diffusion is observed can be estimated to be between the exchange time $\mathrm{t}_{1}=2 \pi / \omega_{\mathrm{e}} \approx 10^{-12} \mathrm{~s}$ and the cut-off time $\mathrm{t}_{2}=2 \pi / \omega_{\mathrm{c}} \approx 10^{-10} \mathrm{~s}$, where we have estimated $\omega_{\mathrm{e}}$ from Eq. (6) with $\mathrm{J}=10 \mathrm{~K}$ and we have estimated $\omega_{\mathrm{c}}$ as an upper limit for the cut-off frequency from the data in Fig. 1. By comparing the results for the two chains, we found that the diffusion constant $\mathrm{D}$ for the Eu-Et chain is two orders of magnitude bigger than for the Gd-Et chain. This is a remarkable result since the exchange constant $\mathrm{J}$ is of the same order of magnitude in the two chains and thus cannot explain the difference. It seems that the spin diffusion in the chain with competing exchange interactions is slowed down. A theoretical investigation of high temperature spin dynamics in similar 1D Heisenberg chains with different magnetic ions would be of great interest.

Regarding the molecular rings, we found that the spin dynamics are dominated by cut-off effects indicating that the systems chosen are not very suitable to investigate spin dynamics, because the intra-molecular anisotropy is too large. In fact, the cut-off time estimated from the experimental results in Fig. 2 is $t_{2}=2 \pi / \omega_{c} \approx 10^{-12} \mathrm{~s}$, which is of the same order of magnitude as the exchange time $t_{1}=2 \pi / \omega_{\mathrm{e}}$. In this case, the data can be fitted almost as well by Eq. (4) or Eq. (7) since the behavior for the two models is similar for large cut-off fields $\mathrm{H}_{\mathrm{c}}$. Nevertheless, the plot of the NSLR in $\mathrm{Cr}_{8} \mathrm{Zn}$ shown in Fig. 2 seems to indicate that there may be a narrow field (frequency) range, in which spin diffusion can be detected. Thus, in order to investigate spin diffusion one should: (i) perform experiments in closed rings and in finite spin segments, as here, but with magnetic ions having a smaller anisotropic interaction energy, like, e.g., $\mathrm{Mn}^{2+}$; and (ii) be sure that the exchange constant $\mathrm{J} / \mathrm{k}_{\mathrm{B}}$ is of the order of $10 \mathrm{~K}$ or more so that the exchange frequency is sufficiently large to observe spin diffusion after a few magnetic lattice steps.

\section{ACKNOWLEDGMENTS}

Funding for this research was obtained from the Italian FIRB Project "RINAME" No. RBAP114AMK and Italian FIRB Project "Futuro in Ricerca" No. RBFR12RPD1.

${ }^{1}$ R. J. Glauber, J. Math. Phys. 4, 294 (1963).

${ }^{2}$ F. Carboni and M. Richards, Phys. Rev. 177, 889 (1969).

${ }^{3}$ D. G. McFadden, R. A. Tahir-Kheli, and G. Bruce Taggart, Phys. Rev. 185, 854 (1969).

${ }^{4}$ F. B. McLean and M. Blume, Phys. Rev. B 7, 1149 (1973).

${ }^{5}$ G. Müller, Phys. Rev. Lett. 60, 2785 (1988).

${ }^{6}$ J. P. Boucher et al., Phys. Rev. B 13, 4098 (1976)

${ }^{7}$ J. Ajiro et al., J. Phys. Soc. Jpn. 44, 420 (1978).

${ }^{8}$ M. Nechtschein et al., Phys. Rev. Lett. 44, 356 (1980).

${ }^{9}$ N. Fujiwara et al., Phys. Rev. 55, R11945 (1997).

${ }^{10}$ M. Takigawa et al., Phys. Rev. Lett. 76, 2173 (1996).

${ }^{11}$ D. Gatteschi, R. Sessoli, and J. Villain, Molecular Nanomagnets (Oxford University Press, Oxford, 2006); A. Müller et al., ChemPhysChem 2, 517 (2001).

${ }^{12}$ J. Villain, J. Phys. 38, 385 (1977).

${ }^{13}$ C. Benelli et al., Inorg. Chem. 28, 272 (1989).

${ }^{14}$ M. Affronte et al., Phys. Rev. B 59, 6282 (1999).

${ }^{15}$ T. Moriya, Prog. Theor. Phys. 16, 23 (1956); 28, 371 (1962).

${ }^{16}$ J. Tang, S. N. Dikshit, and J. R. Norris, J. Chem. Phys. 103, 2873 (1995).

${ }^{17}$ J. H. Luscombe, M. Luban, and F. Borsa, J. Chem. Phys. 108, 7266 (1998).

${ }^{18}$ A. Lascialfari et al., Phys. Rev. B 57, 1115 (1998).

${ }^{19}$ F. Borsa, A. Lascialfari, and Y. Furukawa, in Novel NMR and EPR Techniques, edited by J. Dolinsek, M. Vilfan, and S. Zumer (Springer, New York, 2006), pp. 304-355.

${ }^{20}$ H. Amiri et al., Phys. Rev. B 82, 144421 (2010).

${ }^{21}$ A. Bianchi et al., Phys. Rev. B 79, 144422 (2009). 